\title{
Analytical multikinks in smooth potentials
}

\author{
G. P. de Brito, ${ }^{*}$ R. A. C. Correa, ${ }^{\dagger}$ and A. de Souza Dutra \\ UNESP-Campus de Guaratinguetá-DFQ, Avenida Dr. Ariberto Pereira Cunha, \\ 333 C.P. 205 12516-410 Guaratinguetá, SP, Brazil \\ (Received 9 October 2013; published 28 March 2014)
}

\begin{abstract}
In this work we present an approach that can be systematically used to construct nonlinear systems possessing analytical multikink profile configurations. In contrast with previous approaches to the problem, we are able to do it by using field potentials that are considerably smoother than the ones of the doubly quadratic family of potentials. This is done without losing the capacity of writing exact analytical solutions. The resulting field configurations can be applied to the study of problems from condensed matter to braneworld scenarios.
\end{abstract}

DOI: $10.1103 /$ PhysRevD.89.065039

PACS numbers: 11.10.Kk, 03.65.Sq, 04.20.Jb

\section{INTRODUCTION}

The study of nonlinear systems has been growing in popularity since the 1960s [1,2]. Nowadays nonlinearity is found in many areas of physics, including condensed matter physics, field theory, cosmology, and others [3-24]. Particularly, whenever we have a potential with two or more degenerate minima, one can find different vacua at different portions of the space. Thus, one can find domain walls connecting such regions, and this necessarily leads to the appearance of multikink configurations.

Some time ago, A. Champney and collaborators [25] made an analysis of the reasons for the appearance of multikinks in dispersive nonlinear systems. In part this was motivated by the discovery by Peyrard and Kruskal [26] that a single kink becomes unstable when it moves in a discrete lattice at sufficiently large velocity, whereas multikinks are stable. The effect was shown to be associated with a resonant interaction between the kink and the radiation [27], and the resonances were already observed experimentally [28]. In fact, in an earlier work by Manton and Merabet [29], the mechanism of production of kinks from excitations of the internal mode was discussed in a study of the dynamics of the interaction of two kinks and one antikink in a $\phi^{4}$ model. Furthermore, in a recent work by M. A. Garcia-Ñustes and J. A. González [30], it was shown that a pair of kinklike solitons is emitted during the process of kink breakup by internal mode instabilities in a sine-Gordon model. The multikinks are responsible, for instance, for a mobility hysteresis in a damped driven commensurable chain of atoms [31]. Moreover in arrays of Josephson junctions, instabilities of fast kinks generate bunched fluxon states presenting multikink profiles [32].

In a different context, by working with space-timedependent field configurations, Coleman and collaborators $[33,34]$ analyzed the "fate of the false vacuum" through a

\footnotetext{
"gustavopazzini@gmail.com fis04132@gmail.com

‡dutra@feg.unesp.br
}

semiclassical analysis of an asymmetric $\lambda \phi^{4}$-like model. There, they considered the decaying process of the field configuration from the local to the global vacuum of the model. Moreover, some recent works report fluctuating bouncing solutions $[35,36]$ in models presenting local and global minima.

In all the above physical situations an analytical description of multikinks would be very welcome. However, as far as we know, there is no result in the literature that presents analytical multikink profiles beyond the case of two, the so-called double kink [37], [38], [39]. Here, we intend to fill this gap by presenting a general procedure in order to construct analytical solutions for multikinks, considering reasonably smooth field potentials.

In fact, the idea here is to improve another one used previously in some works dealing with the so-called double-quadratic (DQ) model [40], [41-44], whose potential is given by

$$
V(\phi)=\frac{1}{2} \phi^{2}-|\phi|+\frac{1}{2},
$$

and some of their generalizations like the asymmetrical double-quadratic model (ADQ) [43] and the generalized asymmetrical double-quadratic model (GADQ) [36]. This last one has the advantage that, having the previously mentioned potentials as its limits, it can be used to study systems in which the curvature of the potential is different in each side of the discontinuity point. Moreover, the vacua of the model can be chosen to represent a kind of slow-roll potential, giving rise to inflaton fields that are important in cosmological inflationary scenarios. In fact, a similar model was used to study wet surfactant mixtures of oil and water [45]. The model is such that

$$
V\left(\phi_{\mathrm{GADQ}}\right)= \begin{cases}\lambda\left[\left(\phi_{\mathrm{GADQ}}-\phi_{2}\right)^{2}+V_{2},\right. & \phi \geq 0 \\ \lambda\left(\frac{\phi_{2}^{2}+V_{2}}{\phi_{1}^{2}+V_{1}}\right)\left[\left(\phi_{\mathrm{GADQ}}+\phi_{1}\right)^{2}+V_{1}\right], & \phi \leq 0\end{cases}
$$



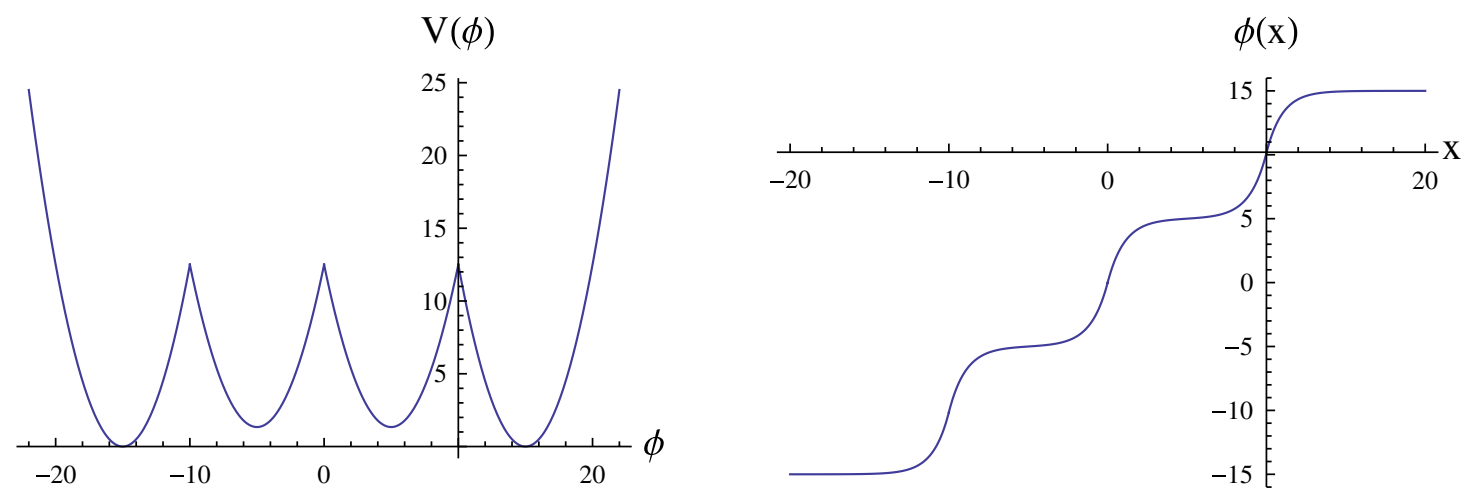

FIG. 1 (color online). Stepwise quadratic potential presenting two global and two local vacua and the corresponding triple kink $\left(a=10, b_{1}=b_{4}=0, \lambda_{1}=1\right.$, and $\left.b_{2}=b_{3}=3\right)$.

where $\lambda, \phi_{1}, \phi_{2}, V_{1}$, and $V_{2}$ are constant parameters that obey the following restrictions:

$$
\phi_{2}>0, \quad \phi_{1}>0, \quad V_{2}>-\phi_{2}^{2} \text { and } V_{1}>-\phi_{1}^{2} \text {. }
$$

In this line of analysis, one can go further by studying a model with a potential for the scalar field presenting four degenerate minima, for instance. This model is written as

$$
V(\phi)= \begin{cases}\frac{\lambda_{1}}{2}\left[\left(\phi+\frac{3 a}{2}\right)^{2}+b_{1}\right] ; & -\infty<\phi \leq-a, \\ \frac{\lambda_{2}}{2}\left(\frac{a^{2}+4 b_{1}}{a^{2}+4 b_{2}}\right)\left[\left(\phi+\frac{a}{2}\right)^{2}+b_{2}\right] ; & -a \leq \phi \leq 0, \\ \frac{\lambda_{3}}{2}\left(\frac{a^{2}+4 b_{1}}{a^{2}+4 b_{3}}\right)\left[\left(\phi-\frac{a}{2}\right)^{2}+b_{3}\right] ; & 0 \leq \phi \leq a, \\ \frac{\lambda_{4}}{2}\left(\frac{a^{2}+4 b_{1}}{a^{2}+4 b_{4}}\right)\left[\left(\phi-\frac{3 a}{2}\right)^{2}+b_{4}\right] ; & a \leq \phi<\infty,\end{cases}
$$

where

$$
\begin{aligned}
& \lambda_{2}=\lambda_{1}\left(\frac{a^{2}+4 b_{1}}{a^{2}+4 b_{2}}\right), \\
& \lambda_{3}=\lambda_{1}\left(\frac{a^{2}+4 b_{1}}{a^{2}+4 b_{3}}\right), \\
& \lambda_{4}=\lambda_{1}\left(\frac{a^{2}+4 b_{1}}{a^{2}+4 b_{4}}\right) .
\end{aligned}
$$

Notice that, despite the fact that the potential is continuous by parts, their physically acceptable solutions must be continuous for both the field and for its first derivative. This restriction comes from the fact that one should seek configurations where the energy density is continuous and nonsingular. Thus one must impose that the solutions are such that $\phi(x)$ and $d \phi(x) / d x$ are continuous throughout the spatial axis. In fact, in this class of potentials, one only needs to require that $\left(\frac{d \Phi}{d x}\right)^{2}$ be continuous due to the continuity of the energy density. In Fig. 1 we can see the profile of the above potential, as well as its triple-kink configuration.
By following the approach developed in [36], one can determine a solution that presents four regions, where the fields are connecting the different (local or global) vacua of the model. Specifically, we look for a solution that is continuous and whose first spatial derivative is also continuous, where $\phi^{(1)}(x) \rightarrow-3 a / 2$ at $x \rightarrow-\infty$ and $\phi^{(4)}(x) \rightarrow 3 a / 2$ at $x \rightarrow \infty$. After straightforward calculations one can verify that the solution is given by

$$
\phi(x)= \begin{cases}-\frac{3 a}{2}+B_{1} e^{x \sqrt{\lambda_{1}}} & -\infty<x<-a, \\ -\frac{a}{2}+A_{2} e^{-x \sqrt{\lambda_{2}}}+B_{2} e^{x \sqrt{\lambda_{2}}} & -a<x<0, \\ \frac{a}{2}+A_{3} e^{-x \sqrt{\lambda_{3}}}+B_{3} e^{x \sqrt{\lambda_{3}}} & 0<x<a, \\ \frac{3 a}{2}+A_{4} e^{-x \sqrt{\lambda_{4}}} & a<x<\infty,\end{cases}
$$

where $A^{\prime} s$ and $B^{\prime} s$ are integrating constants. In order to ensure the necessary gluing conditions at each junction point, one arrives at the following expressions for the parameters in $\phi(x)$ :

$$
\begin{aligned}
& B_{1}=\frac{a \sqrt{\lambda_{2}} e^{a \sqrt{\lambda_{1}}}\left[2 \cosh \left(\sqrt{\lambda_{2}} a\right)+1\right]}{2\left[\sqrt{\lambda_{2}} \cosh \left(\sqrt{\lambda_{2}} a\right)+\sqrt{\lambda_{1}} \sinh \left(\sqrt{\lambda_{2}} a\right)\right]}, \\
& A_{2}=\frac{a\left[\sqrt{\lambda_{2}}+\sqrt{\lambda_{1}}\left(1+2 e^{-a \sqrt{\lambda_{2}}}\right]\right.}{2\left[\sqrt{\lambda_{2}}+\sqrt{\lambda_{1}}+\left(\sqrt{\lambda_{2}}-\sqrt{\lambda_{1}}\right) e^{-2 a \sqrt{\lambda_{2}}}\right]}, \\
& B_{2}=-\frac{2 a \sqrt{\lambda_{1}}+a\left(\sqrt{\lambda_{1}}-\sqrt{\lambda_{2}}\right) e^{-a \sqrt{\lambda_{2}}}}{4\left[\sqrt{\lambda_{2}} \cosh \left(\sqrt{\lambda_{2}} a\right)+\sqrt{\lambda_{1}} \sinh \left(\sqrt{\lambda_{2}} a\right)\right]}, \\
& A_{3}=\frac{a\left[-\sqrt{\lambda_{3}}+\sqrt{\lambda_{4}}\left(1+2 e^{a \sqrt{\lambda_{3}}}\right]\right.}{2\left[\sqrt{\lambda_{3}}-\sqrt{\lambda_{4}}+\left(\sqrt{\lambda_{4}}+\sqrt{\lambda_{3}}\right) e^{2 a \sqrt{\lambda_{3}}}\right]},
\end{aligned}
$$

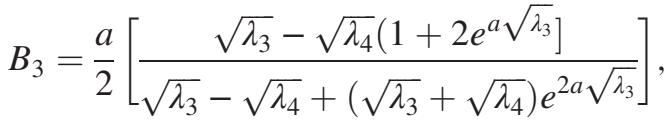

$$
\begin{aligned}
& A_{4}=\frac{-a \sqrt{\lambda_{3}} e^{a \sqrt{\lambda_{4}}}\left[2 \cosh \left(\sqrt{\lambda_{3}} a\right)+1\right]}{2\left[\sqrt{\lambda_{3}} \cosh \left(\sqrt{\lambda_{3}} a\right)+\sqrt{\lambda_{4}} \sinh \left(\sqrt{\lambda_{3}} a\right)\right]} \text {. }
\end{aligned}
$$


However, in all these models one has the potentials which are discontinuous in the first derivative with respect to the field. This is the price to be paid in order to assure that the second order derivatives describing the evolution of the field configuration be linear by parts, and the nonlinear effect comes precisely from that discontinuity.

Notwithstanding, we do not know how to solve analytically only linear equations but some nonlinear too. For instance, we can get exact analytical solutions for the socalled $\lambda \phi^{4}$ and the sine-Gordon models.

The principal idea to be developed in this work is to use this ability, associated with the possibility of constructing a smooth multidegenerate minima potential by gluing the potentials of many $\lambda \phi^{4}$ models, or sine-Gordon and $\lambda \phi^{4}$ models, for different ranges of the scalar field. Once the potential is constructed, one can use an absolutely analogous procedure to that used for the case of the DQ model and his fellows. As a consequence, we develop in this work an approach which allow us to construct models with multistep kinks, due to the presence of a chosen number of intermediate local vacua, in considerably smooth potentials.

\section{MODELS INVOLVING $\boldsymbol{n}$ LOCAL VACUA}

As previously asserted, we present here a general approach capable of describing analytical configurations with an arbitrary number of local vacua and, as a consequence, presenting a multikink profile. In this work we present some models which, as far as we know, are new in the literature and are examples of a wider class of models which can be analytically solved in order to construct field configurations with an arbitrary number of "steps" in a general multikink profile. The potentials that describe these models present two global vacua $\left(V\left(\phi_{M}\right)=0\right)$ and $n$ local ones $\left(V\left(\phi_{m}\right)>0\right)$. As asserted in the Introduction section, they allow one to obtain multikink analytical solutions in smooth potentials.

The first model considered here is the one defined as

$$
V(\phi)= \begin{cases}\frac{\lambda^{2}}{2}\left[a^{2}-(\phi-n b)^{2}\right]^{2} ; & \phi \geq n b \\ \frac{\alpha^{2}}{2} B^{2}-\frac{\alpha^{2}}{2}\left[b^{2}-(\phi-k b)^{2}\right]^{2} ; & (k-1) b \leq \phi<(k+1) b \\ \frac{\lambda^{2}}{2}\left[a^{2}-(\phi+n b)^{2}\right]^{2} ; & \phi<-n b,\end{cases}
$$

where, for even $n, k$ shall assume all odd values inside the interval $[1-n, n-1]$, and for odd values of $n, k$ will assume all the even values along the same interval. In other words, $n$ defines the number of local vacua and $k$ labels each one of them. We will denote the global vacua as $\phi_{M}$, and by $\phi_{k}$ the local ones. Furthermore, the matching points will be denoted by $\phi_{J}$. For the case presented in this section, the global vacua will be localized at the points where $\phi_{M}= \pm(a+n b)$, while the local ones will be found at $\phi_{k}=k b$. In order to get smooth connections, the stepwise potential that we study here was constructed through the junction of polynomial potentials of degree four, the socalled $\phi^{4}$ model, which in each region was conveniently dislocated. In the border regions the shift was given by $\phi \rightarrow \phi \pm n b$, while in the intermediate regions $[(k-1) b \leq$ $\phi<(k+1) b]$, the inverted $\phi^{4}$ model was used. The constant which is added at each intermediate region, is conveniently chosen in order to keep the global vacua at the extremals equal to zero and, as a consequence, we must to impose that $B>b^{2}$ in order grant that these vacua are really local. Since we are dealing with a stepwise potential, some matching conditions must be imposed at the border $\phi_{J}$ of each region. For instance we shall have

$$
\lim _{\phi \rightarrow \phi_{J}^{-}} V(\phi)=\lim _{\phi \rightarrow \phi_{J}^{+}} V(\phi),
$$

which implies in the constraint,

$$
\lambda a^{2}=\alpha B .
$$

It is interesting to note that this relation between $\alpha$ and $\lambda$ is also enough to keep the derivative of the potential continuous at the junctions.

The second model that we will consider in this work is such that one has

$$
V(\phi)= \begin{cases}\frac{\lambda^{2}}{2}\left[a^{2}-\left(\phi-\frac{n \pi}{\mu}\right)^{2}\right]^{2} ; & \phi \geq \frac{n \pi}{\mu} \\ \frac{\alpha^{2}}{2}\left[B^{2}+\frac{1+(-1)^{n} \cos (\mu \phi)}{2}\right] ; & |\phi|<\frac{n \pi}{\mu} \\ \frac{\lambda^{2}}{2}\left[a^{2}-\left(\phi+\frac{n \pi}{\mu}\right)^{2}\right]^{2} ; & \phi \leq \frac{-n \pi}{\mu} .\end{cases}
$$

Once more, $n$ is an integer number corresponding to the number of local vacua of the potential. In this case we construct the model by gluing two $\phi^{4}$ potentials, located at the border regions, with a sine-Gordon potential which will be responsible for the intermediary local vacua. In this model, the global vacua are at $\phi_{M}= \pm(a+n \pi / \mu)$, while the local ones are at the points where $\phi_{k}=k \pi / \mu$. For even $n, k$ assume all the odd numbers along the interval $[1-n, n-1]$, and for odd $n, k$ assume all the even values at the same interval. Now, the continuity condition leads to the following constraint between the coupling constants of the potential

$$
\lambda a^{2}=\alpha \sqrt{1+B^{2}} .
$$


As in the previous model, this condition is also enough to keep the derivative of the potential continuous. As we are going to see below, the relation (12) is capable of granting the continuity of the field configuration, assuring that the energy of the configuration stays finite.

\section{SOLUTION FOR THE FIRST SMOOTH POTENTIAL LOCAL VACUA}

In this section we develop the solutions and analyze some features of the first model here proposed, the one defined in (8). We are primordially interested in solutions connecting the global vacua of the potential. These solutions obey the following boundary conditions:

$$
\lim _{x \rightarrow \pm \infty} \phi(x)= \pm(a+n b) .
$$

Since we are working with the usual Lagrangian density for a self-interacting scalar field, the corresponding equation of motion is

$$
\partial_{\mu} \partial^{\mu} \phi+\frac{d V(\phi)}{d \phi}=0 .
$$

But, we are looking for the static solutions, which can be boosted in order to recover the traveling ones. So, the above equation is simplified to

$$
\phi^{\prime \prime}=\frac{d V(\phi)}{d \phi},
$$

which can be integrated to

$$
\frac{1}{2} \phi^{\prime 2}=V(\phi)
$$

It must be noted that the corresponding integration constant was chosen as zero, since we are interested in configurations that go asymptotically to some vacuum of the field potential.

From Eq. (16), as usual, we can write

$$
\frac{d \phi}{d x}= \pm \sqrt{2 V(\phi)}
$$

and from the above we can conclude that the solution for $\phi(x)$ will be monotonically growing or decreasing according the chosen sign of the right-hand side of the equation. On the other hand, we are interested in solutions going from the negative vacuum to the positive one, so we will look for a field that increases with $x$.

As we have seen before, $V(\phi)$ is a continuous by parts function; specifically, the potential has $n+2$ distinct regions, each one governed by one differential equation coming from (16). So, in order to identify the solutions and their respective regions, we will label them as $\phi_{i}(x)$, with $1 \leq i \leq n+2$ coming from the left to the right.

There is a question that must be carefully treated, and that is the case of the domain of the solutions $\phi(x)$, since each region in the space of the fields corresponds to another in the coordinate space. In order for the analysis to become more precise, we relate each region $i$ of the potential $V(\phi)$ to a set $\Phi_{i}$ such that $\phi_{i}(x) \in \Phi_{i}$. Corresponding to each set $\Phi_{i}$, we have a set $X_{i}$, so that we can associate each element $x \in X_{i}$ to an element of $\Phi_{i}$ through the map $\phi: X_{i} \rightarrow \Phi_{i}$. In fact, each set $\Phi_{i}$ is well determined due to the definition of the potential $V(\phi)$. However, it is still necessary to determine the sets $X_{i}$. For the first region of the potential, $\Phi_{1}=\{\phi \mid-\infty<\phi<-n b\}$, the boundary condition (13) leads to $x \rightarrow-\infty \Rightarrow \phi(x) \rightarrow-(a+n b)$. Furthermore, since $\phi(x)$ is a growing function, there is a certain value of $x_{1}$ such that $\phi\left(x_{1}\right)=-n b$, in such a way that if $x<x_{1}$ then $\phi(x) \in \Phi_{1}$, and for $x \geq x_{1}$ the solution $\phi(x)$ must be in the second region of the potential. Thus, we can conclude that $X_{1}=\left\{x \mid-\infty<x<x_{1}\right\}$. In the second region of the potential, $\Phi_{2}=\{\phi \mid-n b \leq \phi<(2-n) b\}$, we can use the same argument in order to conclude that $X_{2}=\left\{x \mid x_{1} \leq\right.$ $\left.x<x_{2}\right\}$, with $\phi\left(x_{2}\right)=(2-n) b$ and for $x \geq x_{2}$ the solution $\phi$ corresponds to the third region of the potential and so on. Generally speaking, we can write for the intermediary regions of the potential $\Phi_{i}=\{\phi \mid(k-1) b \leq \phi<(k+1) b\}$, and the domain of $\phi(x)$ can be defined as $X_{i}=\left\{x \mid x_{i-1} \leq\right.$ $\left.x<x_{i}\right\}$. Finally, for the right-hand border of the potential we have $\Phi_{n+2}=\{\phi \mid+n b \leq \phi<+\infty\}$, and the domain in the coordinate space will be given by $X_{n+2}=\left\{x \mid x_{n+1} \leq\right.$ $x<+\infty\}$.

Solution in the first region: Here we are interested in solutions like $\phi: X_{1} \rightarrow \Phi_{1}$. In this region the corresponding differential equation is given by

$$
\frac{1}{2} \phi^{\prime 2}=\frac{\lambda^{2}}{2}\left(a^{2}-(\phi+n b)^{2}\right)^{2} .
$$

Performing the translation $\varphi=\phi+n b$, the above equation can be cast in the form

$$
\frac{1}{2} \varphi^{2}=\frac{\lambda^{2}}{2}\left(a^{2}-\varphi^{2}\right)^{2},
$$

and this last equation corresponds to the usual one for the $\varphi^{4}$ model and can be easily integrated to give $\varphi(x)=$ $a \tanh \left(\lambda a\left(x-x_{0}\right)\right)$. Returning to the original variable, one obtains

$$
\phi(x)=a \tanh \left(\lambda a\left(x-x_{1}\right)\right)-n b ; \quad-\infty<x<x_{1},
$$

which satisfies the adopted boundary conditions.

Solution in the region $n+2$ : Focusing our attention in the right border of the potential, where we seek a solution like $\phi: X_{n+2} \rightarrow \Phi_{n+2}$, we can see that 


$$
\frac{1}{2} \phi^{2}=\frac{\lambda^{2}}{2}\left(a^{2}-(\phi-n b)^{2}\right)^{2} .
$$

Again we can perform a displacement as in the above $\varphi=\phi-n b$, and this leads to

$$
\phi(x)=a \tanh \left(\lambda a\left(x-x_{n}\right)\right)+n b ; \quad x_{n+1} \leq x<+\infty .
$$

Once more the boundary conditions are respected as required.

Solutions in the intermediary regions: Let us now deal with the case where $\phi: X_{i} \rightarrow \Phi_{i}$. In those regions, the differential equations are given by

$$
\frac{1}{2} \phi^{\prime 2}=\frac{\alpha^{2}}{2} B^{2}-\frac{\alpha^{2}}{2}\left[b^{2}-(\phi-k b)^{2}\right]^{2},
$$

and, in this case, we need a bit more manipulation before getting the solution. First of all, we rewrite the equation in the form

$$
\frac{d \varphi}{d x}=\alpha \sqrt{\left(A-\varphi^{2}\right)\left(\varphi^{2}-\tilde{A}\right)}
$$

where $A=b^{2}+B, \tilde{A}=b^{2}-B$ and $\varphi=\phi-k b$. Now, making the transformation $\varphi=-\sqrt{A} \cos \theta$, it can be rewritten as

$$
\frac{d \theta}{d x}=\sqrt{\frac{2}{B}} \lambda a^{2} \sqrt{1-m \sin ^{2} \theta},
$$

where we used Eq. (10) and defined $m=\frac{A}{A-\tilde{A}}=\frac{b^{2}+B}{2 B}$. Then, integrating it between $x_{i-1}$ and $x$ we get

$$
\begin{aligned}
\int_{\theta\left(x_{i-1}\right)}^{\theta(x)} \frac{d \theta^{\prime}}{\sqrt{1-m \sin ^{2} \theta^{\prime}}} & =\sqrt{\frac{2}{B}} \lambda a^{2} \int_{x_{i-1}}^{x} d x^{\prime} \\
& =\sqrt{\frac{2}{B}} \lambda a^{2}\left(x-x_{i-1}\right) .
\end{aligned}
$$

The left-hand side of (26) can be identified with an elliptical integral and, provided that $m \in[0,1]$, it can be solved in terms of Jacobi elliptic functions [46]. More precisely we have

$$
\int_{\theta\left(x_{i-1}\right)}^{\theta(x)} \frac{d \theta^{\prime}}{\sqrt{1-m \sin ^{2} \theta^{\prime}}}=\left.\operatorname{sn}^{-1}(\sin \theta \mid m)\right|_{\theta\left(x_{i-1}\right)} ^{\theta(x)} .
$$

Substituting this result in (26), and after some manipulation, we arrive at

$$
\begin{aligned}
\sin \theta(x)= & \operatorname{sn}\left(\sin ^{-1}\left[\sin \theta\left(x_{i-1}\right)\right]\right. \\
& \left.+\sqrt{\frac{2}{B}} \lambda a^{2}\left(x-x_{i-1}\right) \mid \frac{b^{2}+B}{2 B}\right) .
\end{aligned}
$$

Finally, returning to the original variables we get

$$
\begin{gathered}
\phi(x)=\sqrt{b^{2}+B} \mathrm{cn}\left(\delta_{i-1}+\sqrt{\frac{2}{B}} \lambda a^{2}\left(x-x_{i-1}\right) \mid \frac{b^{2}+B}{2 B}\right)+k b ; \\
x_{i-1} \leq x<x_{i},
\end{gathered}
$$

where $\operatorname{sn}(u \mid m)$ represents the elliptic sine function, $\mathrm{cn}(u \mid m)$ represents the elliptic cosine, and we defined that $\delta_{i-1}=\mathrm{sn}^{-1}\left[\sin \theta\left(x_{i-1}\right)\right]$.

Now, using the relation $\phi-k b=-\sqrt{A} \cos \theta$ and also the continuity conditions at the junction points, which are given by $\phi\left(x_{i-1}\right)=(k-1) b$, it can be verified that $\delta_{i-1}$ must satisfy the following constraint equation:

$$
\operatorname{cn}\left(\delta_{i-1} \mid \frac{b^{2}+B}{2 B}\right)+\frac{b}{\sqrt{b^{2}+B}}=0 .
$$

It can be verified from above that $\delta_{i-1}$ is independent from the chosen region of the potential, which allow us to use the notation $\delta \equiv \delta_{i-1}$. However, from Eq. (30) we can conclude that $\delta$ cannot be univocally determined, since there is an infinite number of solutions for $\delta$ due to the periodicity of the elliptic cosine. On the other hand, despite the fact that the solutions of (30) are enough to keep the continuity of $\phi(x)$, not all of them grant the continuity of its derivative at the junction points. Furthermore, it can be numerically observed that half of the roots of (30) lead to continuous $\phi(x)$, while the other half lead to continuous $\phi^{\prime}(x)$, and this behavior under the continuity of the solutions is alternate. Fortunately, it can be numerically verified that the first negative root of Eq. (30) is able to grant the continuity of $\phi(x)$ and its derivative. A further important point is the one related to the determination of the position of the junction points $x_{i}$. In fact, $x_{1}$ can be arbitrarily chosen due to the translation symmetry inherent to the model. However, the points $x_{2}, x_{3}, \ldots, x_{i}$ depend of the choice of $x_{1}$. Once more using the boundary conditions at the junction points, we get the constraint relation

$$
\operatorname{cn}\left(\delta+\sqrt{\frac{2}{B}} \lambda a^{2}\left(x_{i}-x_{i-1}\right) \mid \frac{b^{2}+B}{2 B}\right)=\frac{b}{\sqrt{b^{2}+B}} .
$$

Once $x_{1}$ is specified, we can use the above equation in order to determine $x_{2}$. It can be seen from the above equation that $x_{i}-x_{i-1}$ is independent of the region of the potential that is under study, so we can verify that 

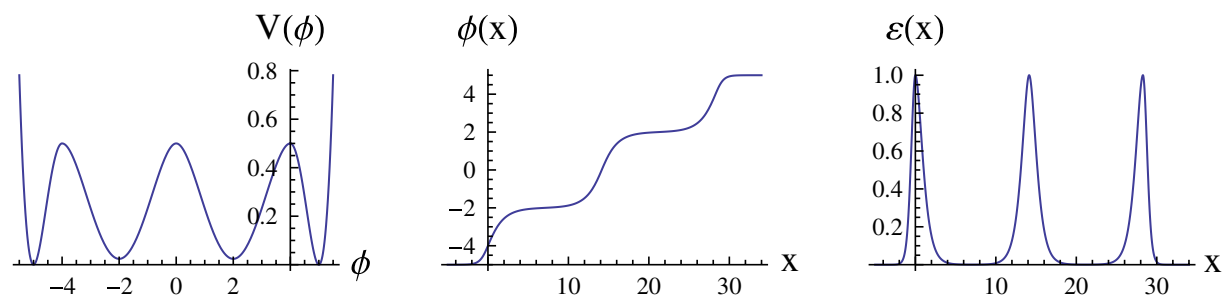

FIG. 2 (color online). Smooth symmetric potential presenting two global and two local vacua, its triple kink, and energy density $(a=\lambda=1, n=2, b=2$, and $B=4.1)$.

$$
\begin{aligned}
x_{2}-x_{1} & =x_{3}-x_{2}=x_{4}-x_{3}=\cdots \\
& =x_{i}-x_{i-1}=\cdots \\
& =x_{n+1}-x_{n} .
\end{aligned}
$$

By using this last equation, we can still conclude that one can write

$$
x_{i}=(i-1) x_{2}-(i-2) x_{1}
$$

so, given a $x_{1}$, the remaining $x_{i}$ can be computed from Eqs. (31) and (33).

In general we can write the solution of this model in the form

$$
\phi(x)= \begin{cases}a \tanh \left(\lambda a\left(x-x_{1}\right)\right)-n b ; & -\infty<x<x_{1} \\ \sqrt{b^{2}+B} \operatorname{cn}\left(\delta+\sqrt{\frac{2}{B}} \lambda a^{2}\left(x-x_{i-1}\right) \mid \frac{b^{2}+B}{2 B}\right)+k b ; & x_{i-1} \leq x<x_{i} \\ a \tanh \left(\lambda a\left(x-x_{n+1}\right)\right)+n b ; & x_{n+1} \leq x<+\infty .\end{cases}
$$

As it was mentioned above, the parameter $B$ controls the height of the local vacua in the model and, as one can verify, when $B$ approaches its critical value $b^{2}$, the intermediary solutions assume a kinklike profile, and the complete solution behaves like a kind of multikink, like a ladder with many steps, and this, as far as we know, is new in terms of an analytical configuration. In the limit case where $B=b^{2}$, we find a configuration where the potential exhibits only global vacua and, as a consequence, the solution connects the adjacent vacua through kinklike configurations. The field configurations which obey Eq. (16) have their energy density given by

$$
\varepsilon[\phi(x)]=\left(\frac{d \phi(x)}{d x}\right)^{2}
$$

and this leads to the following energy density for the present case

$$
\varepsilon[\phi(x)]= \begin{cases}\lambda^{2} a^{4} \operatorname{sech}^{2}\left(\lambda a\left(x-x_{1}\right)\right) ; & -\infty<x<x_{1} \\ \frac{2 \lambda^{2} a^{4}}{B}\left(b^{2}+B\right) \operatorname{cn}^{2}\left(h(x) \mid \frac{b^{2}+B}{2 B}\right) \operatorname{dn}^{2}\left(h(x) \mid \frac{b^{2}+B}{2 B}\right) ; & x_{i-1} \leq x<x_{i} \\ \lambda^{2} a^{4} \operatorname{sech}^{2}\left(\lambda a\left(x-x_{n+1}\right)\right) ; & x_{n+1} \leq x<+\infty\end{cases}
$$

where $h(x)=\delta+\sqrt{2 / B} \lambda a^{2}\left(x-x_{i-1}\right)$ and $\operatorname{dn}(u, m)$ is a Jacobi function. In fact, the energy density above obtained is an integrable function which keeps the total energy finite as necessary. In Fig. 2, it is presented a typical example of this first situation, including the potential, the corresponding triple kink and its energy density.

\section{ASYMMETRIC VERSION OF A MODEL WITH LOCAL VACUA}

In this section we work with an asymmetrical potential presenting two local vacua and which can be described by the stepwise function

$$
V(\phi)= \begin{cases}\frac{\lambda_{1}^{2}}{2}\left[a_{1}^{2}-\left(\phi-2 b_{1}\right)^{2}\right]^{2} ; & \phi \geq 2 b_{1} \\ \frac{\alpha_{1}^{2}}{2} B_{1}^{2}-\frac{\alpha_{1}^{2}}{2}\left[b_{1}^{2}-\left(\phi-b_{1}\right)^{2}\right]^{2} ; & 0 \leq \phi<2 b_{1} \\ \frac{\alpha_{2}^{2}}{2} B_{2}^{2}-\frac{\alpha_{2}^{2}}{2}\left[b_{2}^{2}-\left(\phi+b_{2}\right)^{2}\right]^{2} ; & -2 b_{2} \leq \phi<0 \\ \frac{\lambda_{2}^{2}}{2}\left[a_{2}^{2}-\left(\phi+2 b_{2}\right)^{2}\right]^{2} ; & \phi<-2 b_{2} .\end{cases}
$$

This model was constructed from the potential (8), However, for the sake of simplicity, we considered a version with only two local vacua, but it is important to remark that it can be extended in order to have an arbitrary 
number of local vacua. Thus, like in the symmetric case, we must to keep it continuous at the junction points and, using Eq. (9), we get the following constraint between their coupling constants

$$
\lambda_{1} a_{1}^{2}=\lambda_{2} a_{2}^{2}=\alpha_{1} B_{1}=\alpha_{2} B_{2} .
$$

One can verify that the conditions $B_{1}>b_{1}^{2}$ and $B_{2}>b_{2}^{2}$ are also necessary to grant the presence of local vacua. The solution of the differential equation (16) in each region of the potential (37) follows the same line of reasoning as in the previous case and, due to this, we will present the solution directly in this section. So, in this case we have

$$
\phi(x)= \begin{cases}a_{1} \tanh \left(\lambda_{1} a_{1}\left(x-x_{1}\right)\right)-2 b_{1} ; & -\infty<x<x_{1} \\ \sqrt{b_{1}^{2}+B_{1}} \operatorname{cn}\left(\delta_{1}+\sqrt{\frac{2}{B_{1}}} \lambda_{1} a_{1}^{2}\left(x-x_{1}\right) \mid \frac{b_{1}^{2}+B_{1}}{2 B_{1}}\right)-b_{1} ; & x_{1} \leq x<x_{2} \\ \sqrt{b_{2}^{2}+B_{2}} \operatorname{cn}\left(\delta_{2}+\sqrt{\frac{2}{B_{2}}} \lambda_{1} a_{1}^{2}\left(x-x_{2}\right) \mid \frac{b_{2}^{2}+B_{2}}{2 B_{2}}\right)+b_{2} ; & x_{2} \leq x<x_{3} \\ a_{2} \tanh \left(\lambda_{1} \frac{a_{1}^{2}}{a_{2}}\left(x-x_{3}\right)\right)+2 b_{2} ; & x_{3} \leq x<+\infty .\end{cases}
$$

The continuity of $\phi(x)$, makes necessary that the constants $\delta_{1}$ and $\delta_{2}$ satisfy the following conditions

$$
\begin{aligned}
& \operatorname{cn}\left(\delta_{1} \mid \frac{b_{1}^{2}+B_{1}}{2 B_{1}}\right)+\frac{b_{1}}{\sqrt{b_{1}^{2}+B_{1}}}=0, \\
& \operatorname{cn}\left(\delta_{2} \mid \frac{b_{2}^{2}+B_{2}}{2 B_{2}}\right)+\frac{b_{2}}{\sqrt{b_{2}^{2}+B_{2}}}=0 .
\end{aligned}
$$

As it can be seen, in contrast with the case of Eq. (30), in this case the $\delta$ parameters depend on the region of the potential. Furthermore, as in the previous case, there are many solutions for $\delta$ satisfying the above equations. Once more it can be checked numerically that the first negative solutions warrant the continuity of $\phi(x)$ and its derivative. We can also see how $x_{1}, x_{2}$ and $x_{3}$ are related to each other. As in the previous case $x_{1}$ can be arbitrarily chosen and, once it is defined, the remaining coordinates, $x_{2}$ and $x_{3}$, can be obtained through the equations

$$
\begin{aligned}
& \operatorname{cn}\left(\delta_{1}+\sqrt{\frac{2}{B_{1}}} \lambda_{1} a_{1}^{2}\left(x_{2}-x_{1}\right) \mid \frac{b_{1}^{2}+B_{1}}{2 B_{1}}\right)=\frac{b_{1}}{\sqrt{b_{1}^{2}+B_{1}}}, \\
& \operatorname{cn}\left(\delta_{2}+\sqrt{\frac{2}{B_{2}}} \lambda_{1} a_{1}^{2}\left(x_{3}-x_{2}\right) \mid \frac{b_{2}^{2}+B_{2}}{2 B_{2}}\right)=\frac{b_{2}}{\sqrt{b_{2}^{2}+B_{2}}} .
\end{aligned}
$$

Infact, each one of the above equations is very similar to the one in (31), but in the asymmetrical case there is no expression which is analogous to (32).

In this model, the parameters which are responsible for controlling the local vacua are $B_{1}$ and $B_{2}$, and when they approach respectively their critical values $b_{1}^{2}$ and $b_{2}^{2}$, the intermediary solutions start to present the expected typical kink profile. In the particular case where $n=2$, the solution obtained is an asymmetrical triple kink, as one can see in Fig 3. Finally, through Eq. (35) it can be computed the energy density, which is given by

$$
\varepsilon[\phi(x)]= \begin{cases}\lambda_{1}^{2} a_{1}^{4} \operatorname{sech}^{2}\left(\lambda_{1} \mathrm{a}_{1}\left(\mathrm{x}-\mathrm{x}_{1}\right)\right) ; & -\infty<x<x_{1} \\ \frac{2 \lambda_{1}^{2} a_{1}^{4}}{B_{1}}\left(b_{1}^{2}+B_{1}\right) \mathrm{cn}^{2}\left(h_{1}(x) \mid \frac{b_{1}^{2}+B_{1}}{2 B_{1}}\right) \operatorname{dn}^{2}\left(h_{1}(x) \mid \frac{b_{1}^{2}+B_{1}}{2 B_{1}}\right) ; & x_{1} \leq x<x_{2} \\ \frac{2 \lambda_{1}^{2} a_{1}^{4}}{B_{2}}\left(b_{2}^{2}+B_{2}\right) \operatorname{cn}^{2}\left(h_{2}(x) \mid \frac{b_{2}^{2}+B_{2}}{2 B_{2}}\right) \operatorname{dn}^{2}\left(h_{2}(x) \mid \frac{b_{2}^{2}+B_{2}}{2 B_{2}}\right) ; & x_{2} \leq x<x_{3} \\ \lambda_{1}^{2} a_{1}^{4} \operatorname{sech}^{2}\left(\lambda_{1} \frac{a_{1}^{2}}{a_{2}}\left(x-x_{3}\right)\right) ; & x_{3} \leq x<+\infty,\end{cases}
$$
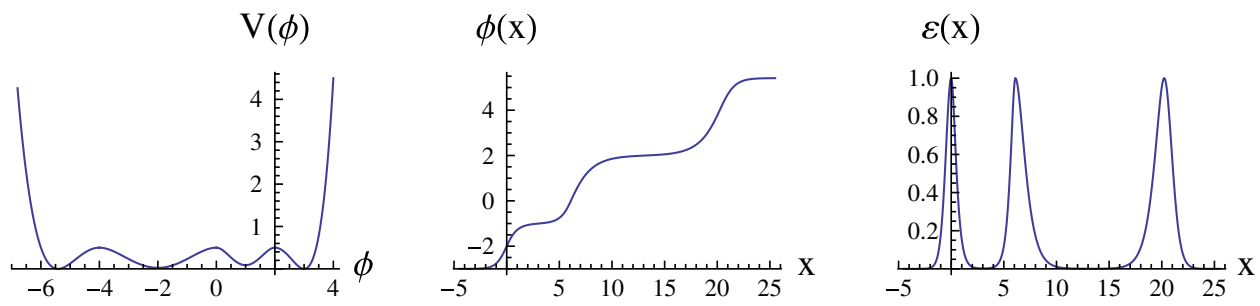

FIG. 3 (color online). Smooth asymmetric potential presenting two global and two local vacua, its triple kink, and energy density $\left(a_{1}=\lambda_{1}=b_{1}=1, b_{2}=2, \lambda_{2}=1 / 2, a_{2}=\sqrt{2}, B_{1}=1.1\right.$, and $\left.B_{2}=4.2\right)$. 
where we defined $h_{1}(x)=\delta_{1}+\sqrt{2 / B_{1}} \lambda_{1} a_{1}^{2}\left(x-x_{1}\right)$ and $h_{2}(x)=\delta_{2}+\sqrt{2 / B_{2}} \lambda_{1} a_{1}^{2}\left(x-x_{2}\right)$.

\section{SOLUTION OF THE SECOND SMOOTH MODEL WITH SYMMETRIC LOCAL VACUA}

Now, we will treat the case of the potential (11). We are again interested in solutions connecting a negative vacuum with a positive one, so that they shall obey the following boundary conditions

$$
\lim _{x \rightarrow \pm \infty} \phi(x)= \pm(a+n \pi / \mu)
$$

and using the very same argument of the first model, one is lead to conclude that $\phi(x)$ shall be a monotonically increasing function. Despite the fact that $V(\phi)$ is written in terms of only three regions, it will be convenient to divide the axis $\phi$ in $2 n+2$ regions, each one localized between a local maximum and the next adjacent maxima. In each potential region we associate a set $\Phi_{i}$, and this leads us to

$$
\begin{aligned}
\Phi_{1} & =\{\phi \mid \phi<-n \pi / \mu\}, \\
\Phi_{2} & =\{\phi \mid-n \pi / \mu \leq \phi<(1-n) \pi / \mu\}, \\
\vdots & \\
\Phi_{i} & =\{\phi \mid(i-n-2) \pi / \mu \leq \phi<(i-n-1) \pi / \mu\}, \\
\vdots & \\
\Phi_{2 n+2} & =\{\phi \mid n \pi / \mu \leq \phi\} .
\end{aligned}
$$

As it was done in the case of the first model, we associate a set $X_{i}$ to each $\Phi_{i}$, and we get that $\phi: X_{i} \rightarrow \Phi_{i}$. This allow us to define the sets $X$ as

$$
\begin{aligned}
X_{1} & =\left\{x \mid x_{1}>x\right\}, \\
X_{2} & =\left\{x \mid x_{1} \leq x<x_{2}\right\}, \\
& \vdots \\
X_{i} & =\left\{x \mid x_{i-1} \leq x<x_{i}\right\}, \\
& \vdots \\
X_{2 n+2} & =\left\{x \mid x_{2 n+1} \leq x\right\},
\end{aligned}
$$

where $\phi\left(x_{i}\right)=(i-n-1) \pi / \mu$.

Solution in region 1 : In this region the solution $\phi: X_{1} \rightarrow \Phi_{1}$, comes from the equation

$$
\frac{1}{2} \phi^{\prime 2}=\frac{\lambda^{2}}{2}\left(a^{2}-(\phi+n \pi / \mu)^{2}\right)^{2} .
$$

We can identify the above equation with the one appearing in (18) simply by doing $b=\pi / \mu$ and $\phi(x)$ can be obtained from (20), giving

$$
\phi(x)=a \tanh \left(\lambda a\left(x-x_{1}\right)\right)-n \pi / \mu ; \quad-\infty<x<x_{1} .
$$

It is easy to verify that it obeys the correct boundary conditions.

Solution in the $2 n+2$ region: In this last region $\phi: X_{2 n+2} \rightarrow \Phi_{2 n+2}$ the differential equation is such that

$$
\frac{1}{2} \phi^{\prime 2}=\frac{\lambda^{2}}{2}\left(a^{2}-(\phi-n \pi / \mu)^{2}\right)^{2} .
$$

Now, choosing $b=\pi / \mu$, the solution of (22) leads us to

$$
\phi(x)=a \tanh \left(\lambda a\left(x-x_{2}\right)\right)+n \pi / \mu ; \quad x_{2} \leq x<+\infty .
$$

Intermediary solutions: In these regions the differential equations look like

$$
\frac{1}{2} \phi^{\prime 2}=\frac{\alpha^{2}}{2}\left[B^{2}+\frac{1+(-1)^{n} \cos (\mu \phi)}{2}\right] .
$$

Again, we are looking for a solution of the type $\phi(x): X_{i} \rightarrow \Phi_{i}$. However, the process of solution will be the same in all the intermediary regions, and it will be necessary only to adjust the appropriate boundary conditions in each of them. First of all, we will work with an even $n$. In this case, we can rewrite the differential equation (50) as

$$
\frac{d \phi}{d x}=\alpha \sqrt{B^{2}+1-\sin ^{2}(\mu \phi / 2)} .
$$

Performing the transformation of variable $\varphi=\mu \phi / 2$, one can integrate the above equation in the following manner,

$$
\int_{\varphi\left(x_{i-1}\right)}^{\varphi(x)} \frac{d \varphi^{\prime}}{\sqrt{1-m \sin ^{2} \varphi^{\prime}}}=\frac{\mu \lambda a^{2}}{2} \int_{x_{i-1}}^{x} d x^{\prime}=\frac{\mu \lambda a^{2}}{2}\left(x-x_{i-1}\right),
$$

where we defined $m \equiv 1 /\left(1+B^{2}\right)$ (with $m \in[0,1]$ ) and used Eq. (12) in order to eliminate the parameter $\alpha$ in terms of $\lambda a^{2}$. The left-hand side of this last equation represents an elliptic integral, and (27) allows us to write

$$
\sin (\mu \phi(x) / 2)=\operatorname{sn}\left(\delta_{i}+\frac{\mu \lambda a^{2}}{2}\left(x-x_{i-1}\right) \mid \frac{1}{1+B^{2}}\right),
$$

where $\delta_{i}=\operatorname{sn}^{-1}\left[\sin \left(\mu \phi\left(x_{i-1}\right) / 2\right)\right]$. On the other hand, by using $\phi\left(x_{i}\right)=(i-n-1) \pi / \mu$, it can be shown that

$$
\sin \left(\mu \phi\left(x_{i-1}\right) / 2\right)=(-1)^{\frac{n}{2}+1} \sin (i \pi / 2) .
$$

At this point we shall be careful, since the field $\phi(x)$ depends on the function $\arcsin (x)$ which, by its turn, must 
have its image and dominion very well specified, due its multivalence. In order to avoid such kind of problem, we specify

$$
\arcsin :[-1,1] \rightarrow[-\pi / 2,+\pi / 2] .
$$

On the other hand, the boundary conditions are assured since Eq. (51) is invariant under transformations of the type $\phi(x) \rightarrow \phi(x)+k \pi$ (valid for integer values of $k$ ). Thus, we can write

$\phi(x)=\frac{2}{\mu} \arcsin \left[\operatorname{sn}\left(\delta_{i}+\frac{\mu \lambda a^{2}}{2}\left(x-x_{i-1}\right) \mid \frac{1}{1+B^{2}}\right)\right]-\frac{2 k \pi}{\mu} ;$ $x_{i-1} \leq x<x_{i}$.

Now, using the boundary condition $\phi\left(x_{i}\right)=(i-n-$ 1) $\pi / \mu$ in the above equation, we get

$\phi\left(x_{i-1}\right)=\frac{2}{\mu} \arcsin \left[\operatorname{sn}\left(\delta_{i} \mid \frac{1}{1+B^{2}}\right)\right]-\frac{2 k \pi}{\mu}=\frac{i-n-2}{\mu} \pi$.

Using the definition of $\delta_{i}$ and Eq. (54), we can conclude that

$$
k= \begin{cases}\frac{n+2-i}{2} ; & \text { for even } i \\ \frac{n+2+\left[(-1)^{\frac{n}{2}+1}-1\right] i}{2} ; & \text { for odd } i .\end{cases}
$$

Before finishing the discussion of this section, we shall determine the relative position of the $x_{i}$. From the boundary condition in $\phi\left(x_{i}\right)$, we can write

$$
\begin{aligned}
\phi\left(x_{i}\right) & =\frac{2}{\mu} \arcsin \left[\operatorname{sn}\left(\delta_{i}+\frac{\mu \lambda a^{2}}{2}\left(x_{i}-x_{i-1}\right) \mid \frac{1}{1+B^{2}}\right)\right]-\frac{2 k \pi}{\mu} \\
& =\frac{i-n-1}{\mu} \pi .
\end{aligned}
$$

From the definition of $\delta_{i}$ and using Eq. (58), we arrive at

$$
\begin{aligned}
& \operatorname{sn}\left(\frac{\mu \lambda a^{2}}{2}\left(x_{i}-x_{i-1}\right) \mid \frac{1}{1+B^{2}}\right)=1 ; \quad \text { for even } i, \\
& \delta_{i}+\frac{\mu \lambda a^{2}}{2}\left(x_{i}-x_{i-1}\right)=0 ; \quad \text { for odd } i .
\end{aligned}
$$

Through the above equation it can be shown that

$x_{3}-x_{1}=x_{5}-x_{3}=x_{7}-x_{7}=\cdots=x_{i}-x_{i-2} ;$

for even $i$,

$x_{4}-x_{2}=x_{6}-x_{4}=x_{8}-x_{6}=\cdots=x_{i}-x_{i-2} ;$

for odd $i$.

Again, once the value of $x_{1}$ is defined, we can determine $x_{2}$ and $x_{3}$ through (60) and the remaining junction points are given by the above equations.

In general, the solution for the multikink field configuration of this model can be written in the form

$$
\phi(x)= \begin{cases}a \tanh \left(\lambda a\left(x-x_{1}\right)\right)-n \pi / \mu ; & -\infty<x<x_{1} \\ \frac{2}{\mu} \arcsin \left[\operatorname{sn}\left(\delta_{i}+\frac{\mu \lambda a^{2}}{2}\left(x-x_{i-1}\right) \mid \frac{1}{1+B^{2}}\right)\right]-\frac{2 k \pi}{\mu} ; & x_{i-1} \leq x<x_{i} \\ a \tanh \left(\lambda a\left(x-x_{2 n+2}\right)\right)+n \pi / \mu ; & x_{2 n+2} \leq x<+\infty .\end{cases}
$$

As expected, this solution has the same profile as the one obtained for the first model which is appearing in Fig. 2. All the above discussion was done for the case with even $n$. However, the same procedure can be used for the case with odd $n$ and the result will have the same appearance, and due to this we will not present the details here. The energy density of this soliton can also be calculated by using Eq. (35), and it presents a very similar profile of the one corresponding to the first model, which can be seen in Fig. 2.

\section{TOPOLOGICAL PROPERTIES}

In this section we will consider some topological properties for the models proposed in this paper. It is well know that the usual kinklike configurations are topological solutions and, as a consequence, we may use some index in order to classify such solutions with respect to the topological features. The so-called topological charge is an example of the topological index often used in the literature. Here, we show that the topological charge is well defined for the models presented here, and we will calculate this charge for both models. We have defined a topological current in the usual way [17], namely,

$$
j^{\mu}=\varepsilon^{\mu \nu} \partial_{\nu} \phi
$$

where $\varepsilon^{\mu \nu}$ is the Levi-Civita symbol. It is not difficult to conclude that a conservation law follows from the definition of $j^{\mu}$, in fact we have $\partial_{\mu} j^{\mu}=0$ as a consequence of the antisymmetry of $\varepsilon^{\mu \nu}$. Note that both the topological charge and its conservation law are well defined, since we have ensured that first and the second derivatives of the scalar fields solutions are continuous at any point. The topological charge may be defined in terms of $j^{\mu}$ in the following way: 


$$
Q=\int_{-\infty}^{\infty} d x j^{0} .
$$

Note that $j^{0}=\varepsilon^{0 \nu} \partial_{\nu} \phi=\partial \phi / \partial x$; thus, after integration we obtain the following result:

$$
Q=\phi(x=+\infty)-\phi(x=-\infty) .
$$

For the first model considered in this work, whose solution is given by Eq. (34), we have the asymptotic values for the scalar field given by

$$
\phi(x=+\infty)=a+n b \quad \text { and } \quad \phi(x=-\infty)=-a-n b .
$$

Therefore, the topological charge for the first model is given by

$$
Q=2 a+2 n b .
$$

It is interesting to note that the topological charge obtained for the usual $\phi^{4}$ theory [which may be obtained with $n=0$ in Eq. (8)] is given by $Q_{0}=2 a$; thus, we may rewrite the case with arbitrary $n$ as follows

$$
Q_{n}=Q_{0}+2 n b \text {. }
$$

Repeating the same procedure for the second model, whose solution is given by Eq. (62), we obtain $\phi(x=$ $+\infty)=a+n \pi / \mu$ and $\phi(x=-\infty)=-a-n \pi / \mu$. Therefore, in this case the topological charge is given by

$$
Q_{n}=2 a+2 n \pi / \mu=Q_{0}+2 n \pi / \mu .
$$

We may note that in both cases the topological charge increases linearly with the number of local vacua as well as with the separation of them.

\section{CONCLUSIONS}

In this work, we have introduced a method that can be systematically used to obtain analytical multikink configurations which come from very smooth stepwise scalar field potentials. The approach was presented through three examples, and their corresponding typical triple kink and energy density profiles can be seen in Figs. 2 and 3. In fact, as it can be observed from the case studied in the Introduction section, these multikink profiles can be constructed in the case of potentials similar to the DQ model and its generalizations (see Fig. 1). However, those potentials present discontinuity in their derivative at the junction points, which do not happen in the cases we have introduced in this work. Among the possible applications of our results, we are presently interested in the possibility of constructing multi-braneworld scenarios.

\section{ACKNOWLEDGMENTS}

The authors thank the CNPq and FAPESP for partial financial support.
[1] G. B. Whitham, Linear and Non-Linear Waves, (Wiley, New York, 1974).

[2] A. C. Scott, F. Y. F. Chiu, and D. W. Mclaughlin, Proc. IEEE 61, 1443 (1973).

[3] R. Rajaraman and E. J. Weinberg, Phys. Rev. D 11, 2950 (1975).

[4] H. Arodz, Phys. Rev. D 52, 1082 (1995); Nucl. Phys. B450, 174 (1995); H. Arodz and A. L. Larsen, Phys. Rev. D 49, 4154 (1994).

[5] A. Strumia and N. Tetradis, Nucl. Phys. B542, 719 (1999).

[6] C. Csaki, J. Erlich, C. Grojean, and T. J. Hollowood, Nucl. Phys. B584, 359 (2000).

[7] M. Gremm, Phys. Lett. B 478, 434 (2000).

[8] A. de Souza Dutra and A. C. Amaro de Faria, Jr., Phys. Rev. D 72, 087701 (2005).

[9] M. A. Shifman and M. B. Voloshin, Phys. Rev. D 57, 2590 (1998).

[10] D. Bazeia, M. J. dos Santos, and R. F. Ribeiro, Phys. Lett. A 208, 84 (1995); D. Bazeia, W. Freire, L. Losano, and R. F. Ribeiro, Mod. Phys. Lett. A 17, 1945 (2002).
[11] A. Campos, Phys. Rev. Lett. 88, 141602 (2002).

[12] A. Melfo, N. Pantoja, and A. Skirzewski, Phys. Rev. D 67, 105003 (2003).

[13] A. de Souza Dutra, Phys. Lett. B 626, 249 (2005).

[14] A. de Souza Dutra and A. C. Amaro de Faria, Jr., Phys. Lett. B 642, 274 (2006).

[15] V. I. Afonso, D. Bazeia, and L. Losano, Phys. Lett. B 634, 526 (2006).

[16] M. Giovannini, Phys. Rev. D 75, 064023 (2007); 74087505 (2006).

[17] R. Rajaraman, Solitons and Instantons (North-Holland, Amsterdam, 1982).

[18] A. Vilenkin and E. P. S. Shellard, Cosmic Strings and Other Topological Defects (Cambridge University, Cambridge, England, 1994).

[19] M. Cvetic and H. H. Soleng, Phys. Rep. 282, 159 (1997).

[20] T. Vachaspati, Kinks and Domain Walls: An Introduction to Classical and Quantum Solitons (Cambridge University, Cambridge, England, 2006).

[21] R. Rajaraman, Phys. Rev. Lett. 42, 200 (1979).

[22] L. J. Boya and J. Casahorran, Phys. Rev. A 39, 4298 (1989). 
[23] A. de Souza Dutra, A. C. Amaro de Faria, Jr., and M. Hott, Phys. Rev. D 78, 043526 (2008).

[24] M. K. Prasad and C. M. Sommerfield, Phys. Rev. Lett. 35, 760 (1975); E. B. Bolgomol'nyi, Sov. J. Nucl. Phys. 24, 449 (1976).

[25] A. Champneys and Y. S. Kivshar, Phys. Rev.E61, 2551 (2000).

[26] M. Peyrard and M. Kruskal, Physica (Amsterdam) 14D, 88 (1984).

[27] See, e.g., A. V. Ustinov, M. Cirillo, and B. A. Malomed, Phys. Rev. B 47, 8357 (1993).

[28] H. S. J. van der Zant, T. P. Orlando, S. Watanabe, and S. H. Strogatz, Phys. Rev. Lett. 74, 174 (1995).

[29] N. S. Manton and H. Merabet, Nonlinearity 10, 3 (1997).

[30] M. A. Garcia-Ñustes and J. A. González, Phys. Rev. E 86, 066602 (2012).

[31] O. M. Braun, T. Dauxois, M. V. Paliy, and M. Peyrard, Phys. Rev. Lett. 78,1295 (1997); O. M. Braun, A. R. Bishop, and J. Röder, ibid. 79, 3692 (1997).

[32] A. V. Ustinov, B. A. Malomed, and S. Sakai, Phys. Rev. B 57, 11691 (1998).

[33] S. Coleman, Phys. Rev. D 15, 2929 (1977).

[34] Curtis G. Callan, Jr. and S. Coleman, Phys. Rev. D 16, 1762 (1977).
[35] G. V. Dunne and Q. H. Wang, Phys. Rev. D 74, 024018 (2006).

[36] A. de Souza Dutra and R. A. C. Correa, Phys. Lett. B 679, 138 (2009).

[37] D. Bazeia, J. Menezes, and R. Menezes, Phys. Rev. Lett. 91, 241601 (2003).

[38] A. de Souza Dutra, Physica D (Amsterdam) 238, 798 (2009).

[39] A. E. R. Chumbes and M. B. Hott, Phys. Rev. D 81, 045008 (2010).

[40] B. Horovitz, J. A. Krumhansl, and E. Domany, Phys. Rev. Lett. 38, 778 (1977).

[41] S. E. Trullinger and R. M. DeLeonardis, Phys. Rev. A 20, 2225 (1979).

[42] H. Takayama and K. Maki, Phys. Rev. B 20, 5009 (1979).

[43] Stavros Theodorakis, Phys. Rev. D 60, 125004 (1999).

[44] D. Bazeia, A. S. Inácio, and L. Losano, Int. J. Mod. Phys. A 19, 575 (2004).

[45] G. Gompper and S. Z. Zschocke, Phys. Rev. A 46, 4836 (1992).

[46] M. Abramowitz and I. A. Stegun, Handbook of Mathematical Functions with Formulas, Graphs, and Mathematical Tables (Dover, New York, 1972). 